\title{
Non-Empirical Metrics for Ontology Visualizations Evaluation and Comparing ${ }^{\star}$
}

\author{
Ildar Baimuratov ${ }^{[0000-0002-6573-131 X]}$ and Than Nguyen ${ }^{[0000-0002-6679-7839]}$ \\ ITMO University, Kronverksky Pr. 49, bldg. A, St. Petersburg, 197101, Russia \\ \{baimuratov.i, nguyenngocthan92\}@gmail.com
}

\begin{abstract}
There are numerous ontology visualization systems, however, the choice of a visualization system is non-trivial, as there is no method for evaluation and comparing them, except for empirical experiments, that are subjective and costly. In this research, we aim to develop non-empirical metrics for ontology visualizations evaluation and comparing. First, we propose several half-formal metrics that require expert evaluation. These metrics are completeness, semanticity, and conservativeness. We apply the proposed metrics to evaluate and compare VOWL and Logic Graphs visualization systems. And second, we develop a completely computable measure for the complexity of ontology visualizations, based on graph theory and information theory. In particular, ontology visualizations are considered as hypergraphs and the information measure is derived from the Hartley function. The usage of the proposed information measure is exemplified by the evaluation of visualizations of the sample of axioms from the DoCO ontology in Logic Graphs and Graphol. These results can be practically applied for choosing ontology visualization systems in general and regarding a particular ontology.
\end{abstract}

Keywords: Ontology Visualization, Expert Evaluation, Hypergraphs, Information Measure.

\section{Introduction}

Visualization of an ontology improves comprehension of knowledge it contains. There are numerous ontology visualization systems, the reviews are presented in [1-3]. However, the choice of a visualization system is non-trivial, as there is no method for evaluation and comparing of ontology visualization systems present at the literature, except for empirical experiments, that are subjective and costly.

Little researches consider the evaluation of visualization systems in general. In [4] the authors propose some recommendations considering graph diagrams, like minimization of crossings between edges. The authors of [5] present empirical research on applying these criteria to automatic graph layout algorithms. In [6] several new shapebased metrics are proposed for large graphs. All these metrics are based on empirical experiments, i.e. on human assessments.

Copyright $\odot 2020$ for this paper by its authors. Use permitted under Creative Commons License Attribution 4.0 International (CC BY 4.0).

* Publication is supported by RFBR grant 20-01-00358 


\section{I. Baimuratov and Than N.}

Therefore, we aim to develop formal metrics for ontology visualizations estimation. 'Formal' means that they must be objective and computable. Some metrics we propose require external knowledge of the language being visualized, its semantics, and knowledge of other visualization systems, therefore, they are half-formal and require expert evaluation. Another criterion is based on graph theory and information theory and is fully computable.

The outline of the paper is as follows: in Section 2 we propose several metrics for expert evaluation and in Section 3 we derive the information measure for ontology visualizations complexity.

\section{Expert evaluation}

First, we propose to consider several features of visualization systems that, though related to the formal properties, like completeness, still require expert evaluation, as they involve external knowledge.

\subsection{Completeness}

The most important property of a visualization system is its completeness with respect to the language being visualized, because if a visualization system can not represent some axioms of an ontology, the system can not be applied to the ontology. In addition, a common reference language serves as a common denominator for comparing different visualization systems.

Ontologies are denoted on the OWL language [7]. The OWL 1 standard provided three increasingly expressive sub-languages: OWL Lite, OWL DL, and OWL Full. In this paper, we consider OWL DL language, as it provides the maximum expressiveness, retaining decidability.

The formal foundation of OWL is description logics (DLs) [8]. DLs are a family of logic languages, that can be used to represent the terminological knowledge of an application domain. We consider axioms, formulated with the SHOIN description logic syntax, as it corresponds to OWL DL language. We evaluate completeness of a visualization system by counting the number of SHOIN syntax entities that the system can represent.

\subsection{Semanticity}

We suppose, the advantage of a visualization with respect to a reference language is that it improves comprehension of a formula with representing its semantic. Therefore, we propose to evaluate the ability of a visualization system to represent semantics of expressions.

We consider a diagram of a visualization system for a logical relation as semantical, if it represents the semantic of the relation. For example, compare the visualization of conjunction from Graphol [9], Fig. 1, with the corresponding Venn diagram [10], Fig. 2. Venn diagram represents that these two sets have common elements, while in Graphol conjunction is just labeled with a hexagon. 


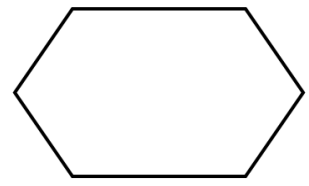

Fig. 1. Conjunction in Graphol

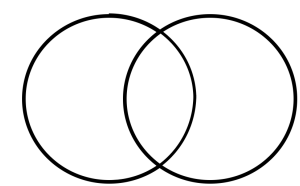

Fig. 2. Conjunction in Venn diagrams

\subsection{Conservativeness}

Finally, we suppose that it is important to use existing graphic primitives from mathematical theories, as in the other case, i.e. introducing new graphic primitives, instead of helping a user to understand an ontology it forces him or her to learn just one more language. Considering again the example above, in Graphol a user has to learn that hexagon denotes conjunction, while if the Venn diagram was used, the user familiar with Venn diagrams would have understood the diagram without additional instructions. Therefore, we consider a visualization system as conservative, if it uses the existing graphic primitives.

\subsection{Example of evaluation}

We provide an evaluation of the VOWL [11] visualization system as an example. We examined its completeness with respect to OWL DL language, its semanticity, the ability to represent the semantics of relations, and whether its graphic primitives are new or adopted from common visualization systems. See Table 1.

Here we see that VOWL can represent only 12 of 15 entities of the SHOIN description logic, therefore, its completeness rate is 0.8 . The diagrams for concepts, conjunction, disjunction, and equivalence are semantical and conservative, as they are based on Venn diagrams. The diagram for roles can be considered as graph-theory based, therefore, it is also semantical and conservative. The diagram for negation is conservative, as it uses the sign of negation from logic, but it is not semantical since it doesn't represent the semantic of negation.

In addition, we provide an example of comparing of visualization systems, performed by the authors. We compare VOWL with Logic graphs (LGs) [12], the semantically oriented ontology visualization method, developed by us. The analogous evaluation of the LGs is in Table 2, the scores of VOWL and LGs are presented in Table 3. As wee see, LGs are complete and more semantical and conservative, than VOWL.

\section{Information measuring}

In the previous section, we proposed several metrics for expert-based evaluation. Properties like completeness and conservativeness are important for visualization systems evaluation, but it is hard to imagine that they would be fully computable. Thus, we propose one more approach to ontology visualization systems evaluation intended to be completely formal. This approach is based on information measuring. 
4 I. Baimuratov and Than N.

Table 1. Expert-based evaluation of VOWL

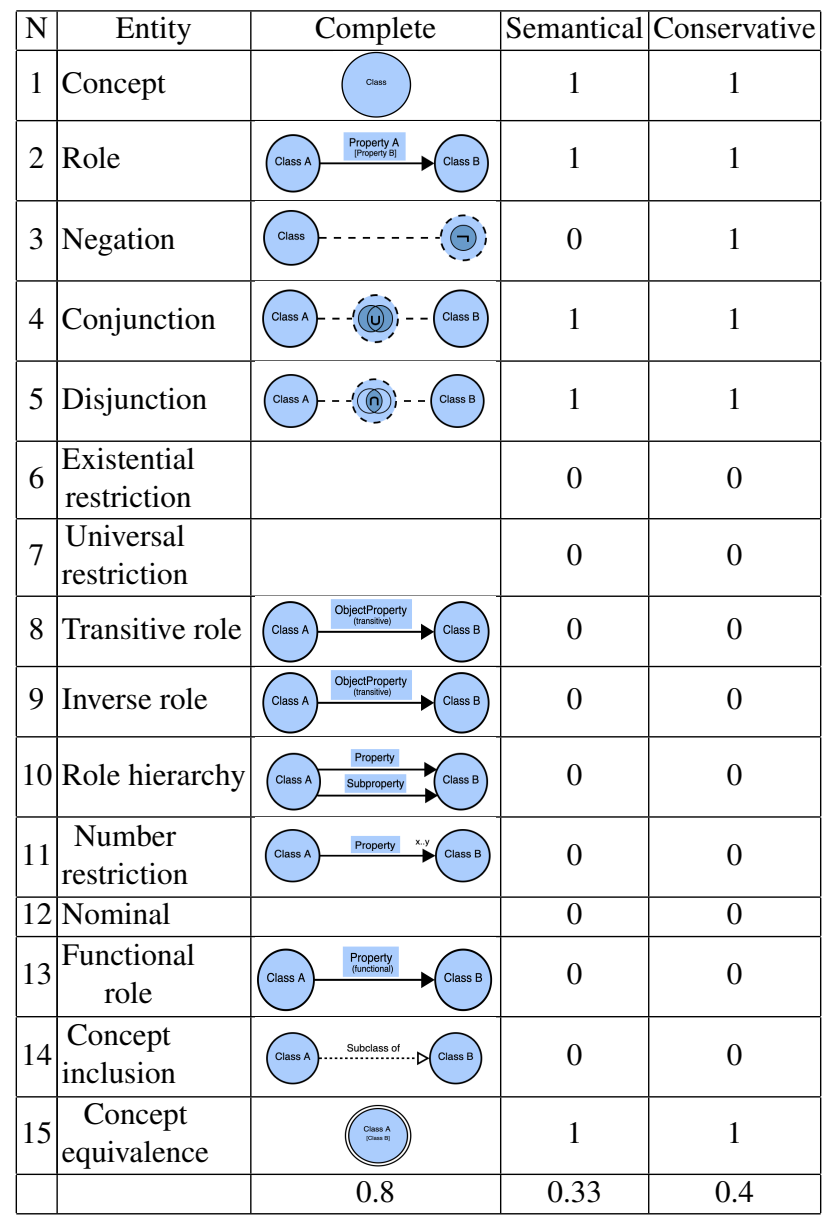

The intended information measure should estimate not the content of the ontology, as it is the same for each visualization, but the complexity of its form. It implies the following requirement for the intended information measure:

- as it should estimate the complexity of an ontology visualization, it should depend on the complexity of its structure, in other words, on the number of nodes, edges, and types of edges;

- it should be normalized, as visualizations of one and the same ontology in different visualization systems can have a different number of nodes and edges;

- as it should measure the visualization complexity, a visualization with a greater number of nodes, edges, or edges types should have a higher value of the measure. 
Non-Empirical Metrics for Ontology Visualizations Evaluation... 5

Table 2. Expert-based evaluation of LGs

\begin{tabular}{|c|c|c|c|c|}
\hline $\mathrm{N}$ & Entity & Complete & Semantical & Conservative \\
\hline 1 & Concept & $\mathrm{C}$ & 1 & 1 \\
\hline 2 & Role & $\mathrm{Cl}$ & 1 & 1 \\
\hline 3 & Negation & & 1 & 1 \\
\hline 4 & Conjunction & $\mathrm{Cl}$ & 1 & 1 \\
\hline 5 & Disjunction & 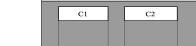 & 1 & 1 \\
\hline 6 & $\begin{array}{l}\text { Existential } \\
\text { restriction }\end{array}$ & \begin{tabular}{|l|l} 
Domain & \\
& \\
&
\end{tabular} & 1 & 1 \\
\hline 7 & $\begin{array}{l}\text { Universal } \\
\text { restriction }\end{array}$ & Domain & 1 & 1 \\
\hline 8 & Transitive role & & 1 & 1 \\
\hline 9 & Inverse role & R- & 1 & 1 \\
\hline 10 & Role hierarchy & f & 1 & 1 \\
\hline 11 & $\begin{array}{c}\text { Number } \\
\text { restriction }\end{array}$ & 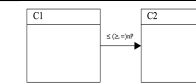 & 0 & 1 \\
\hline 12 & Nominal & at ${ }^{a+a}$ & 1 & 1 \\
\hline 13 & $\begin{array}{c}\text { Functional } \\
\text { role }\end{array}$ & 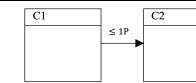 & 0 & 1 \\
\hline 14 & $\begin{array}{l}\text { Concept } \\
\text { inclusion }\end{array}$ & 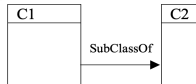 & 0 & 0 \\
\hline 15 & $\begin{array}{c}\text { Concept } \\
\text { equivalence }\end{array}$ & $\mathrm{C}_{2}$ & 1 & 1 \\
\hline & & 1 & 0.8 & 0.93 \\
\hline
\end{tabular}

\subsection{Hypergraphs as the formal framework}

Before defining the information measure, we have to define the formal framework. We propose to consider ontology visualization as a hypergraph. Simple graphs are not suitable for our goals as many ontology visualization systems use edges connecting more than two nodes. A hypergraph can be represented as an incidence matrix, therefore, an ontology visualization can be represented as an incidence matrix as well. 
6 I. Baimuratov and Than N.

Table 3. Expert-based comparing of VOWL and LG

\begin{tabular}{|c|c|c|c|}
\hline & Complete & Semantical & Conservative \\
\hline VOWL & 0.8 & 0.33 & 0.4 \\
\hline LGs & 1 & 0.8 & 0.93 \\
\hline
\end{tabular}

Let there is a hypergraph $H=(X, E)$, where $X$ - is a set of nodes and $E$ is a set of edges. It is represented with $|X| \times|E|$ incidence matrix $A=\left(a_{i j}\right)$, where

$$
a_{i j}=\left\{\begin{array}{l}
1, \text { if } x_{i} \in e_{j} \\
0, \text { otherwise }
\end{array}\right.
$$

for undirected graph and

$$
a_{i j}=\left\{\begin{array}{l}
-1, \text { if }\left(x_{i}, x^{\prime}\right) \in e_{j} \\
1, \text { if }\left(x^{\prime}, x_{i}\right) \in e_{j} \\
0, \text { otherwise }
\end{array}\right.
$$

and

$$
a_{i j}=2 \text { if } e_{j}=\left(x_{i}, x_{i}\right) .
$$

Consider the following axiom from the Document Components Ontology (DoCO) [13] as the example

$$
\text { chapter } \sqsubseteq \exists \text { contains.paragraph } \sqcup \text { section }
$$

and its visualization in the Graphol system, see Fig. 3. Here edges denoting disjunction

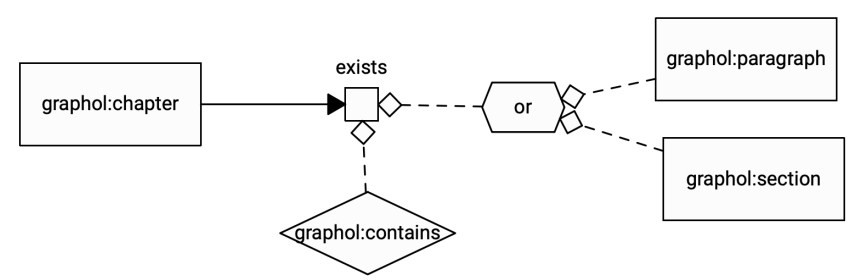

Fig. 3. Visualization of the axiom 1 in Graphol

connect three nodes: 'graphol.paragraph', 'graphol.section' and 'or', therefore, it is the hypergraph. The incidence matrix for this hypergraph is Table 4. Each node of the diagram corresponds to a row of the matrix and each relation - to a column. As nodes 'graphol.paragraph', 'graphol.section' and 'or' are connected with the edge 'or', the corresponding cells have value 1 . In this research, we ignore the direction of edges for simplicity. 
Table 4. The incidence matrix for the axiom 1 in Graphol

\begin{tabular}{|c|c|c|c|}
\hline & subClassOf & graphol.contains & or \\
\hline exists & 1 & 1 & 0 \\
\hline graphol.chapter & 1 & 0 & 0 \\
\hline graphol.contains & 0 & 1 & 0 \\
\hline graphol.paragraph & 0 & 0 & 1 \\
\hline graphol.section & 0 & 0 & 1 \\
\hline or & 0 & 1 & 1 \\
\hline
\end{tabular}

\subsection{The information measure}

There are researches on graph information measuring. The survey of graph entropy measures is in [14]. The authors of [15] perform information-theoretic analysis of edge bundling visualizations in terms of adjacency matrices and mutual information. But none of the measures presented there satisfy our requirement. Therefore, we develop a new graph information measure.

First, we define the set of all different values of an incidence matrix $A$ :

Definition 1. For a given incidence matrix $A$ the set of all values is $\{a\}$.

For undirected graph $\{a\}=\{0,1\}$, for directed $\{a\}=\{-1,0,1\}$, for directed graph with loops $\{a\}=\{-1,0,1,2\}$ and so on.

Now we define the set of all possible edges $\mathcal{E}$ :

Definition 2. For a hypergraph $H$ with a given set of nodes $X$ the set of all possible edges $\mathcal{E}=\{a\}^{X}$.

We are ready to define the information measure for hypergraph complexity estimation by deriving it from the Hartley function [16]

$$
\log _{b}|A|
$$

where $A$ is an arbitrary set and $b$ - an arbitrary number. We substitute the number of edges $|E|$ as $|A|$ and the number of all possible edges $|\mathcal{E}|$ as $b$.

Definition 3. For a hypergraph $H$ with a given set of nodes $X$, a given set of edges $E$ and a set of corresponding incidence matrix values $\{a\}$, the information $I(H)$ is following:

$$
I(H)=\log _{|\mathcal{E}|}|E|=\frac{1}{|X|} \log _{|\{a\}|}|E|
$$

Consider several simple graphs for illustration, see Fig. 4, its information estimation is at the Table 5. As wee see, $H_{2}$ has a more complex structure comparing to $H_{1}$ and, therefore, its information value is higher. $H_{3}$ is directed and each directed edge contains less information, therefore, with the same number of edges its information value is lower compared to $H_{1}$. Summing up, the information measure satisfies the desired properties. 
8 I. Baimuratov and Than N.
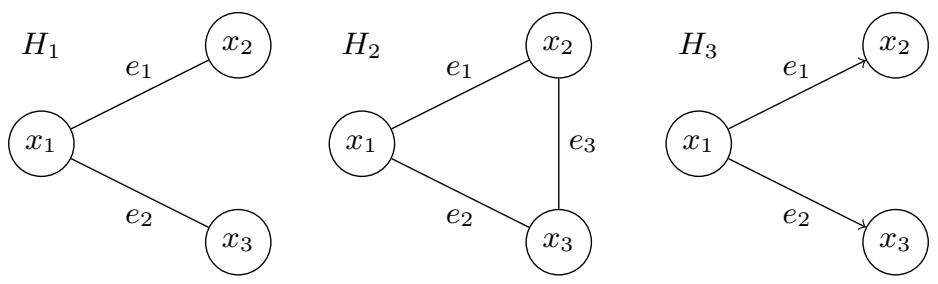

Fig. 4. The graph examples

Table 5. Information estimation of the graph examples

\begin{tabular}{|c|c|c||c|c|}
\hline$H$ & $|X| \mid$ & $|E|$ & $\{a\} \mid$ & $I(H)$ \\
\hline 1 & 3 & 2 & 2 & 0.33 \\
\hline 2 & 3 & 3 & 2 & 0.53 \\
\hline 3 & 3 & 3 & 3 & 0.21 \\
\hline
\end{tabular}

\subsection{Comparing visualizations with the information measure}

We provide an example of comparing ontology visualizations with the developed information measure. Unlike expert-based evaluation, where we compared visualization systems itself, for information measure we have to compare visualizations of a particular ontology. We use the DoCO ontology as it is a real ontology, used in different applications, and it contains nontrivial axioms. We visualized some axioms of this ontology in Graphol and Logic Graphs (LGs) [12], the semantically oriented ontology visualization method, developed by us. The list of axioms and their visualizations are in Table 6.

The example of an incidence matrix for Graphol was provided in Table 4. Now consider the example of the incidence matrix for Logic Graphs. The incidence matrix for the axiom 1 in Logic graphs is Table 7.

We compare LGs with Graphol by measuring information of the corresponding visualizations for the sample of axioms, presented in Table 6. The result is in Table 8. As we see, the average information of LGs on this sample is higher, than of Graphol.

\section{Conclusion}

In this research, we proposed several non-empirical metrics for ontology visualization evaluation and comparing. These metrics are divided into two groups. The first group includes three metrics: completeness, semanticity, and conservativeness. These metrics require expert evaluation and, therefore, they are half-formal. As an example, we compared two ontology visualization systems: VOWL and Logic Graphs.

The second group consists of the completely computable information measure, derived from the Hartley formula, that allows normalized measuring complexity of ontology visualizations, represented as hypergraphs with incidence matrices. As an example, we compared Logic Graphs with Graphol by measuring average information of visualizations of the sample of axioms from the DoCO ontology. 
Non-Empirical Metrics for Ontology Visualizations Evaluation... 9

Table 6. Visualizations of DoCO in LGs and Graphol

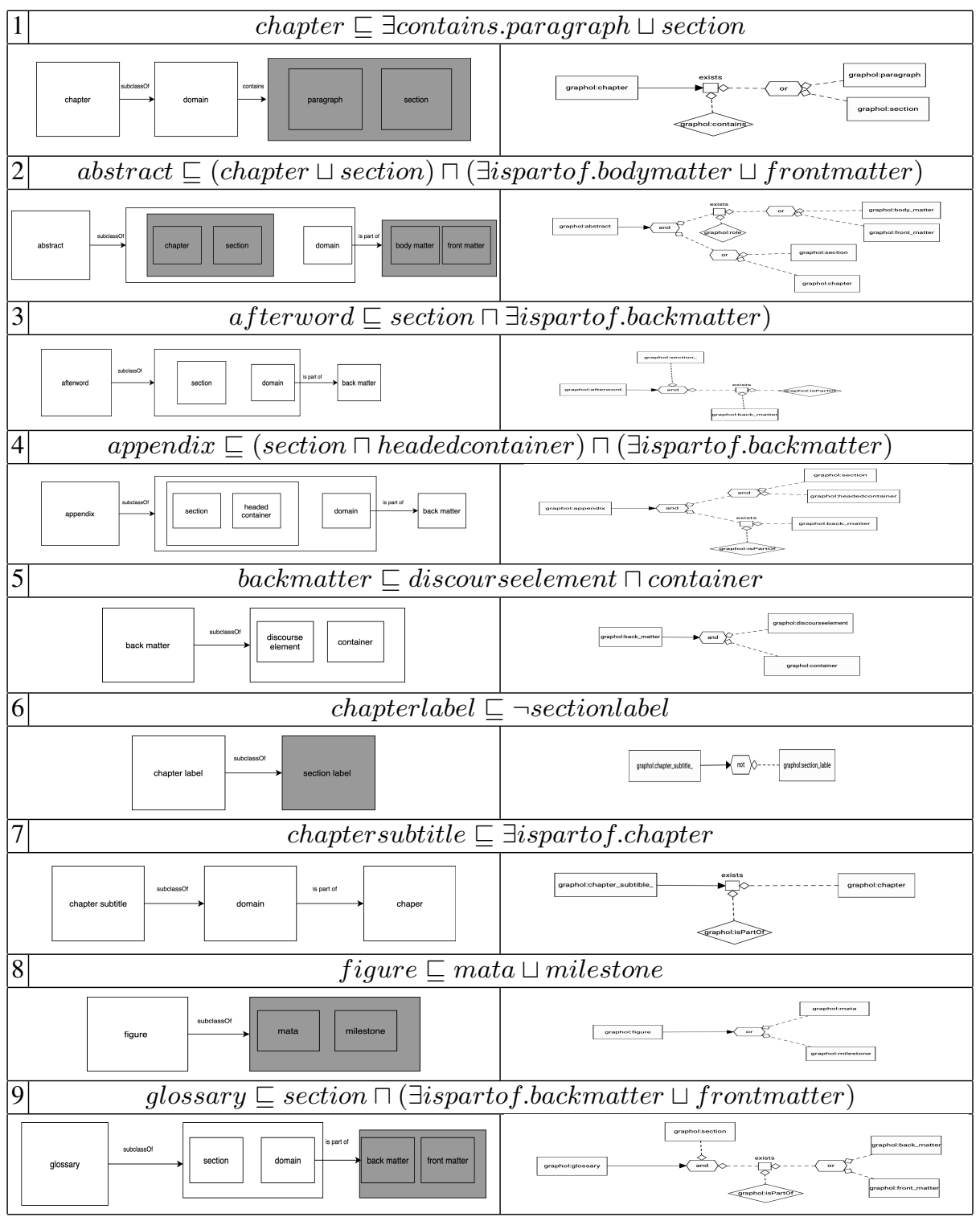

Table 7. The incidence matrix for axiom 1 in LGs

\begin{tabular}{|c|c|c|c|c|c|}
\hline & subClassOf & contains & negation 1 & negation 2 & negation 3 \\
\hline chapter & 1 & 0 & 0 & 0 & 0 \\
\hline conjunction & 0 & 1 & 0 & 0 & 1 \\
\hline domain & 1 & 1 & 0 & 0 & 0 \\
\hline paragraph & 0 & 0 & 1 & 0 & 0 \\
\hline section & 0 & 0 & 0 & 1 & 0 \\
\hline
\end{tabular}


10I. Baimuratov and Than N.

Table 8. Comparing information of ontology visualizations

\begin{tabular}{|c|c|c|c|c|c|c|c|c|}
\hline $\mathrm{N}$ & \multicolumn{4}{|c|}{ LG } & \multicolumn{4}{c|}{ Graphol } \\
\hline & $|X||| E \mid$ & $|\{a\}|$ & $I(H)$ & $|X|$ & $|E|$ & $|\{a\}|$ & $I(H)$ \\
\hline 1 & 5 & 6 & 2 & 0.52 & 6 & 3 & 2 & 0.26 \\
\hline 2 & 9 & 11 & 2 & 0.38 & 10 & 5 & 2 & 0.23 \\
\hline 3 & 5 & 3 & 2 & 0.32 & 6 & 3 & 2 & 0.26 \\
\hline 4 & 7 & 4 & 2 & 0.29 & 8 & 4 & 2 & 0.25 \\
\hline 5 & 4 & 2 & 2 & 0.25 & 4 & 2 & 2 & 0.25 \\
\hline 6 & 2 & 2 & 2 & 0.5 & 3 & 2 & 2 & 0.33 \\
\hline 7 & 3 & 2 & 2 & 0.33 & 4 & 2 & 2 & 0.25 \\
\hline 8 & 4 & 5 & 2 & 0.58 & 4 & 2 & 2 & 0.25 \\
\hline 9 & 7 & 7 & 2 & 0.4 & 8 & 4 & 2 & 0.25 \\
\hline & & & & 0.4 & & & & 0.26 \\
\hline
\end{tabular}

These results can be practically applied for choosing ontology visualization systems in general and regarding a particular ontology. Considering the presented examples, it is recommended to use LGs rather than VOWL in general, as it has higher scores of completeness, semanticity, and conservativeness, and for visualizing the mentioned fragment of the DoCO, as LGs has higher informativeness.

\section{References}

1. Antoniazzi F., Viola F.: RDF graph visualization tools: A survey //2018 23rd Conference of Open Innovations Association (FRUCT).IEEE,25-36 (2018)

2. Dudáš, M.,Lohmann, S., Svátek, V., Pavlov, D.: Ontology visualization methods and tools: A survey of the state of the art. The Knowledge Engineering Review, vol 33, (2018)

3. Steffen, L., Stefan, N. , Florian, H. Thomas, E: Visualizing ontologies with VOWL. Semantic Web. 7. 399-419. (2016)

4. R. Tamassia, C. Batini, and M. Talamo. An algorithm for automatic layout of entityrelationship diagrams. In C. G. Davis, S. Jajodia, P. A. Ng, and R. T. Yeh, editors, 3rd Int. Conf. on Entity-Relationship Approach (ER'83), pages 421-439. North-Holland, 1983.

5. Purchase, Helen \& Carrington, David \& Allder, Jo-Anne. (2002). Empirical Evaluation of Aesthetics-based Graph Layout. Empirical Software Engineering. 7.

6. Eades, Peter \& Hong, Seok-Hee \& Klein, Karsten \& Nguyen, An. (2015). Shape-Based Quality Metrics for Large Graph Visualization. 502-514.

7. Deborah, L., Frank, V.,, et al. Owl web ontology language overview. W3C recommendation, 10(10):2004,(2004)

8. Baader, F.,Calvanese, D. , McGuinness, D. ,Nardi, D. and Patel-Schneider,P.: The Description Logic Handbook: Theory, Implementation, and Applications. Cambridge University Press, second edition, (2007)

9. Graphol official website, Http://www.obdasystems.com/graphol. Last accessed 4 jul 2020

10. Ruskey, F.,Weston, M.:A Survey of Venn Diagrams. The Electronic Journal of Combinatorics, (2005)

11. VOWL official website, Http://vowl.visualdataweb.org/. Last accessed 4 jul 2020

12. Mouromtsev, D.,Baimuratov, I.: Logic Graphs: A complete visualization method for logical languages based on Ch. S. Peirce's existential graphs. CEUR Workshop Proceedings, Vol. 2344, 2019, pp. 1-10. 
13. Ontology DoCO, https://sparontologies.github.io/doco/current/doco.html. Last accessed 4 jul 2020

14. Dehmer, Matthias \& Mowshowitz, Abbe. (2011). A History of Graph Entropy Measures. Information Sciences. 1. 57-78.

15. Wu, Jieting \& Zhu, Feiyu \& Liu, Xin \& Yu, Hongfeng. (2018). An Information-Theoretic Framework for Evaluating Edge Bundling Visualization. Entropy. 20. 625.

16. R. V. L. Hartley, "Transmission of information," in The Bell System Technical Journal, vol. 7, no. 3, pp. 535-563, July 1928. 